AperTO - Archivio Istituzionale Open Access dell'Università di Torino

\title{
IgG4-related nephropathy
}

\section{This is the author's manuscript}

Original Citation:

Availability:

This version is available http://hdl.handle.net/2318/1592769

since 2019-02-13T15:16:38Z

Published version:

DOI:10.1007/s40620-016-0279-4

Terms of use:

Open Access

Anyone can freely access the full text of works made available as "Open Access". Works made available under a Creative Commons license can be used according to the terms and conditions of said license. Use of all other works requires consent of the right holder (author or publisher) if not exempted from copyright protection by the applicable law. 
This is the author's final version of the contribution published as:

Quattrocchio, Giacomo; Roccatello, Dario. IgG4-related nephropathy. JN. JOURNAL OF NEPHROLOGY. 29 (4) pp: 487-493.

DOI: $10.1007 / \mathrm{s} 40620-016-0279-4$

The publisher's version is available at:

http://link.springer.com/content/pdf/10.1007/s40620-016-0279-4

When citing, please refer to the published version.

Link to this full text:

http://hdl.handle.net/2318/1592769 


\title{
IgG4-related nephropathy.
}

Quattrocchio $\mathrm{G}^{1,2}$, Roccatello $\mathrm{D}^{3,4,5}$.

1Nephrology and Dialysis Unit, Giovanni Bosco Hospital, Turin, Italy. g.quattrocchio@libero.it.

2Second level Master in Practical Rheumatology, University of Turin, Turin, Italy. g.quattrocchio@libero.it.

3Nephrology and Dialysis Unit, Giovanni Bosco Hospital, Turin, Italy.

4Department of Rare, Immunologic, Center of Research of Immunopathology and Rare Diseases (CMID), Hematologic and Immunohematologic Diseases, Giovanni Bosco Hospital, Turin, Italy.

5University of Turin, Turin, Italy.

\begin{abstract}
IgG4-related disease (IgG4-RD) is a recently recognized disorder, often with multiple organ involvement, characterized by dense tissue infiltration of IgG4-positive plasma cells, storiform fibrosis, obliterative phlebitis and frequently elevated serum IgG4 concentration. The kidney can be involved either directly or indirectly. The most frequent direct renal manifestations of IgG4-RD are IgG4-related tubulointerstitial nephritis (TIN) and membranous glomerulonephropathy. Retroperitoneal fibrosis (RPF) is another condition that is frequently IgG4-related and that can indirectly affect the kidney causing ureteral obstruction and hydronephrosis. Contrast-enhanced computerized tomography, magnetic resonance imaging and (18)F-fluorodeoxyglucose positron emission tomography/computed tomography show different imaging findings and are useful tools for monitoring therapeutic response. Steroid treatment is the first line of therapy, but relapsing or refractory forms of the disease are frequently observed and require more aggressive therapeutic approaches. At our centre, we treated three cases of aggressive IgG4-related TIN and two cases of IgG4-related RPF with an intensified, immune suppressive protocol, obtaining good results without severe adverse effects.
\end{abstract}

\section{Introduction}

Immunoglobulin (Ig) G4-related disease (IgG4-RD) is a systemic fibroinflammatory immune-mediated condition, identified in the last 15 years, that can mimic a wide range of malignant, infectious and rheumatologic diseases [1-3]. The diagnosis of IgG4-RD unifies many conditions (Table 1) that were previously believed to be confined to single organs and were considered well-defined disorders [3-5]. IgG4-RD can affect virtually any organ system including the meninges, hypophysis, eyes, orbits, salivary and lacrimal glands, thyroid, mediastinum, lungs, pancreas, biliary tree, aorta, lymph nodes, kidney and even nerves, skin and urinary bladder [6].

The hallmark histological features of the disease are a dense lymphoplasmacytic infiltrate enriched with IgG4+ plasma cells, a storiform pattern of fibrosis, and obliterative phlebitis [3, 7]. Radiologic studies, such as ultrasonography (US), computed tomography (CT) or magnetic resonance imaging (MRI), often show tumefactive hypodense lesions, which can be indistinguishable from neoplastic masses [3]. The kidney is involved in approximately $15 \%$ of patients and renal involvement, usually termed IgG4-related kidney disease (IgG4-RKD), may include a wide range of clinical and histological manifestations: tubulointerstitial nephritis (TIN), membranous glomerulonephropathy (MGN) and other infrequent glomerular lesions, pyelitis, and hydronephrosis due to retroperitoneal fibrosis (RPF) [6, 8].

\section{Epidemiology}

The epidemiology of the disease has not been explored in detail. The majority of patients reported in the literature are from Japan, where the estimated prevalence is six cases per 100,000 inhabitants, but the condition has been described all over the world [9]. The average age of disease onset is in the seventh decade of life, and there is a clear male predilection with an overall male:female ratio of $8: 3$, with the exception of the forms involving the head and neck for which the gender ratio seems to be almost equal $[9,10]$.

\section{Pathophysiology}

The pathophysiology of IgG4-RD is poorly understood. Genetic studies have suggested that several human leukocyte antigen (HLA) and non-HLA haplotypes/genotypes are associated with susceptibility to IgG4-RD or to disease relapse after steroid therapy $[3,11]$. Antinuclear antibodies are found in a significant proportion of patients and several 
autoantibodies have been identified, amongst which antibodies against lactoferrin and carbonic anhydrase II are the most frequently detected, but their definitive role in the pathogenesis of the disease is unknown [11]. Some studies have suggested a possible role for molecular mimicry involving Helicobacter pylori (HP), and antibodies targeting HP plasminogen-binding protein (PBP) have been detected in patients with autoimmune pancreatitis, but whether a relationship exists between PBP and IgG4-RD remains to be determined [10,11]. The contribution of innate immune responses to IgG4-RD is increasingly being recognized. In particular, foreign pathogen-associated molecular patterns (PAMPs) and damage-associated molecular patterns (DAMPs) can be recognized by Toll-like receptors and nucleotidebinding oligomerization domain-containing protein 2 (NOD2) in monocytes and basophils, leading to B cell-activating factor (BAFF)-dependent induction of IgG4 production by CD19+ cells [12]. Serum IgG4 levels are frequently elevated. These antibodies possess weak binding properties to complement component $\mathrm{C} 1 \mathrm{q}$ and $\mathrm{Fc}$ receptors; therefore they poorly activate the classic component pathway and participate in antibody-dependent cell-mediated cytotoxicity. Furthermore, because of a process of Fab-arm exchange among different IgG4 molecules, they can form "halfantibodies" with reduced ability to crosslink antigens and form immune complexes. Therefore, IgG4 antibodies are likely to act as suppressors rather than inducers of inflammatory processes [8]. Cellular immunity, particularly $\mathrm{T}$ cells, are implicated in disease pathogenesis. In fact, CD4+ T cells are abundant within IgG4-RD lesions, and tissue T cell cytokines-interleukin (IL)-4, IL-10 and transforming growth factor (TGF)- $\beta$ - are upregulated [13]. A polarized type 1 $\mathrm{T}$ helper or type $2 \mathrm{~T}$ helper cell population might act as a critical mediator of fibrosis by secreting cytokines such as interferon (IFN)- $\gamma$ and TGF- $\beta$, which recruit and activate fibroblasts. A separate $\mathrm{T}$ follicular helper cell population, producing IL-4 and IL-10, might promote class-switching of IgG antibodies to IgG4 and differentiation of B cells into plasma cells $[4,8,10]$. Finally, regulatory T-cells likely play a central role in IgG4 and TGF- $\beta$ production in the interstitium, thus promoting interstitial fibrosis [14].

IgG4 and kidney

Any form of renal involvement in IgG4-RD is collectively referred to as IgG4-related kidney disease and occurs in approximately $15 \%$ of patients $[8,15]$.

\section{IgG4-related tubulointerstitial nephritis (IgG4-related TIN)}

The most common renal manifestation of IgG4-RD is tubulointerstitial nephritis. The majority of data regarding this entity are derived from two biopsy series of a Japanese cohort of 23 patients [16] and an American cohort of 35 patients [17]. Light microscopy demonstrates dense cell interstitial infiltration predominantly composed of plasma cells and lymphocytes that can be either diffuse or multifocal (Fig. 1). Eosinophils are frequently seen. In some cases a dense cellular infiltrate with minimal fibrosis is observed (pattern A), while other cases are characterized by a more densely cellular inflammatory lesion with expansile interstitial fibrosis (pattern B) or collagen-rich, paucicellular fibrosis (pattern C) [17]. Patients with a fibrotic interstitium typically demonstrate the hallmark storiform fibrosis, characterized by a swirling pattern of fibrosis resembling the spokes of a cartwheel with spindle cells radiating from the center [7, 18]. Obliterative phlebitis, a critical pathological feature of $\mathrm{IgG}-\mathrm{RD}$, is rarely seen in IgG4-related TIN, perhaps because of the small size of the specimens obtained by kidney needle biopsy [18]. Mild mononuclear tubulitis is frequently observed [8].

Immunofluorescence studies show focal or diffuse immune complex deposition in the tubular basement membrane (TBM) in more than $80 \%$ of patients, with invariable positive granular staining for IgG, frequent staining for $\mathrm{C} 3$ and occasional staining for C1q [17]. Electron microscopy usually confirms electron dense TBM deposits [17].

Immunostaining of tissue affected by IgG4-related TIN typically reveals more than $10 \mathrm{IgG} 4+$ plasma cells/high-power field (HPF) and an increased IgG4+:IgG+ plasma cell ratio (>40\%) relative to healthy tissue $[8,16]$ (Fig. 2). Two sets of diagnostic criteria for IgG4-related TIN have been proposed by Raissian et al. [17] and Kawano et al. [19], both based on pathological findings, imaging, serology and clinical features.

\section{Glomerular lesions}

Membranous glomerulonephropathy is the most commonly described glomerular disease in IgG4-RKD and has been reported in 7-10\% of patients with IgG4-related TIN [18]. Alexander et al. reported a series of nine patients with MGN-associated IgG4-RD: all subjects presented nephrotic proteinuria, five of them had concomitant TIN, and seven also had IgG4-RD extrarenal involvement; none of the eight examined biopsies showed positivity for the phospholipase A2 receptor [20, 21], suggesting secondary rather than idiopathic concomitant MGN. Other glomerular diseases (Henoch-Schönlein purpura nephritis, IgA nephritis, endocapillary proliferative glomerulonephritis, membranoproliferative glomerulonephritis) have been reported concurrently with IgG4-related TIN or IgG4-RD, but their real relationship with IgG4-RD has yet to be elucidated $[6,18,20]$. 


\section{Retroperitoneal fibrosis (RPF)}

RPF is a chronic inflammatory fibrosing condition that may result from multiple distinct etiologies (infection, radiation therapy, medication, malignancy, trauma) and tends to encase the ureters and adjacent structures causing secondary renal involvement due to hydronephrosis. IgG4-RD is now recognized as the cause of up to two-thirds of cases of idiopathic RPF. Histopathological features such as storiform fibrosis, obliterative phlebitis, and tissue eosinophilia are critical for identifying this disease association (Fig. 3). Extraretroperitoneal manifestations of IgG4-RD are also often present among patients with IgG4-related RPF [22, 23].

\section{Rare manifestations}

IgG4-RKD may cause cystic formations due to severe narrowing and obstruction of the collecting duct [24].

\section{Clinical features}

General symptoms such as fever and malaise are unusual, while organ-specific manifestations due to tumefactive localized or systemic lesions are very frequent $[3,9,10,25,26]$. Nephrologists should suspect IgG4-RKD in male patients aged 60-70 years requiring medical attention for acute or progressive renal failure (TIN), nephrotic range proteinuria (MGN) or back, abdomen and flank pain (RPF), especially in cases with multiorgan manifestations [26].

\section{Laboratory features}

In general, IgG4-RD is characterized by some very frequent, albeit non-specific laboratory abnormalities $[9,26,27]$ : hypergammaglobulinemia ( $80-90 \%$ of cases), elevated (>135 mg/dl) serum IgG4 levels (50-70\%), elevated serum IgE levels (60-70\%), C3 and/or C4 hypocomplementemia (50-70\%), peripheral eosinophilia (35-50\%), antinuclear antibodies (30\%), and rheumatoid factor (20-30\%). In subjects with only slight elevation of serum IgG4 levels, diagnostic specificity may be increased by higher ratios of $\operatorname{IgG} 4$ to total $\operatorname{IgG}(>10 \%)$ or of $\operatorname{IgG} 4$ to IgG1 (>24 \%) [28], even if there are contrasting reports [29]. Elevated circulating plasmoblasts have been observed in active IgG4-RD, even in patients with normal serum IgG4 concentrations, so they could be a potentially useful biomarker for diagnosis, assessment of response to treatment and identification of timing for re-treatment [30]. Compared to general IgG-RD patients, IgG4-RKD patients more frequently show hypocomplementemia and elevated serum IgG4 levels [6, 17, 19, 26]. IgG4-related TIN patients may present progressive renal failure, mild to moderate proteinuria (usually tubular), leukocyturia, and mild hematuria [8, 15-19]. IgG4-related MGN patients present with nephrotic range proteinuria like idiopathic MGN patients, but they lack circulating antibodies against phospholipase A2 receptors, which are found in $70 \%$ of idiopathic MGN [20, 21]. IgG4-related RPF patients may show only mild creatinine elevation or even normal renal function with normal urinalysis [23].

\section{Radiologic features}

Contrast-enhanced CT and MRI play an important diagnostic role both in IgG4-RD and, in particular, in IgG4-RKD [26]. Currently, CT is the primary imaging modality used to examine patients with suspected IgG4-RD [19, 31]. Multiple low-density lesions on enhanced CT are the most common radiologic finding in IgG4-related TIN [6, 19], but diffuse bilateral renal swelling may be observed when severe renal failure is a contraindication for the administration of contrast medium [19]. Kidney abnormalities on CT imaging have been observed in nearly $70 \%$ of patients with IgG4RKD [26]. Retroperitoneal masses with unilateral or bilateral hydronephrosis may be found in IgG4-related RPF [23]. MRI has become a useful imaging method for detecting IgG4-RKD in the early stages. Typical MRI findings are bilateral, multiple, renal parenchymal nodules with $\mathrm{T} 2$ hypointensity, diffusion restriction, and a progressive enhancement pattern. Adding diffusion-weighted imaging (DWI) to conventional MRI may enhance the diagnosis [31] (Fig. 4). Four disease patterns have been described: round or wedge-shaped renal cortical lesions, small (subcentimeter) peripheral cortical nodules, diffuse patchy involvement, and renal pelvis involvement. Renal cortical lesions are usually multiple and bilateral, and are composed of lymphoplasmacytic infiltrates. Focal pyelonephritis or metastases are differential diagnoses if round or wedge-shaped nodules are present, although these can be readily excluded in the appropriate clinical context. Renal involvement in IgG4-related disease rarely manifests as a solitary lesion, which may mimic a neoplasm. Renal lesions are typically hypo-attenuating relative to the native renal parenchyma on contrastenhanced CT images, with mild enhancement seen in the delayed phase images. On MRI, renal lesions appear iso- or hypointense relative to the native renal parenchyma on T1-weighted images, hypointense on T2-weighted images, and demonstrate mild contrast enhancement after contrast medium administration [32]. Some studies have suggested a potential diagnostic role of $18 \mathrm{~F}$-fluorodeoxyglucose (FDG) positron emission tomography/computed tomography 
(PET/CT) for assessing organ involvement, monitoring therapeutic response, and guiding interventional treatment of IgG4-RD [33] (Fig. 5).

\section{Treatment}

Glucocorticoids are the first line of therapy for both IgG4-RD [3, 5, 10, 34] and IgG4-RKD [8, 15, 16, 18].

A starting prednisolone dose of $0.6-1.0 \mathrm{mg} / \mathrm{kg} / \mathrm{daily}$, gradually tapered after $2-4$ weeks depending on the clinical response, is the usual protocol for IgG4-RD patients with organ failure, in particular in cases of type 1 autoimmune pancreatitis, and clinical improvement is frequently rapid, depending on the affected organs and the degree of fibrosis $[3,5,10]$. Indeed, although the initial response is usually good, disease relapse after tapering or discontinuing steroids is very high [10].

Prolonged steroid therapy exposes patients to several potential, adverse side-effects, so traditional disease-modifying antirheumatic drugs (DMARDS) like azathioprine, mycophenolate mofetil, methotrexate and cyclosporine have been used as glucocorticoid-sparing agents even for patients with incomplete response, but their efficacy has not been demonstrated $[5,10]$.

B lymphocyte depletion therapy with rituximab (RTX) has demonstrated efficacy in improving clinical and serologic features of IgG4-RD patients with active inflammation [34-36], even without concomitant steroid therapy [37]. Serial treatments with RTX may lead to progressive decreases in serum IgG4 concentrations and better disease control. Serum IgG4 concentrations may remain low, and clinical disease activity may remain quiescent even after B cell reconstitution in a significant proportion of patients [36].

Steroids are effective for most patients with IgG4-related TIN [16, 17], for some patients with IgG4-related MGN [20], and for some patients with IgG4-related RPF [23]. However, recovery of renal function may not be total. Furthermore, relapse of renal dysfunction following steroid tapering or withdrawal is frequently observed, and irreversible renal failure requiring maintenance hemodialysis may occur, especially in patients with advanced renal damage [8, 18]. Therefore, as shown for IgG4-RD with extrarenal manifestations, B-cell depletion with RTX might yield a more effective and long-lasting response also in IgG4-RKD patients [8, 23].

An international panel of experts very recently developed recommendations for the management of IgG4-RD, confirming glucocorticoids as first-line agents for remission induction in all patients with active, untreated disease (94 $\%$ agreement). On the contrary, there was low agreement (46\%) on the use of steroid-sparing agents, including RTX [38].

At our Center, we treated one patient with IgG4-related TIN with an association therapy of steroids and RTX, and four patients (two with IgG4-related TIN and two with IgG4-related RPF) with the intensified immune suppressive protocol adopted in our Department for severe systemic lupus erythematosus (SLE) nephritis [39] according to the following scheme: three pulses of $15 \mathrm{mg} / \mathrm{kg}$ intravenous (i.v.) methylprednisolone followed by oral tapering prednisone + two pulses of $500 \mathrm{mg}$ i.v. cyclophosphamide (on days 1 and 15) + 4 weekly rituximab administrations (375 mg/m2). Two more doses of rituximab were administered 1 and 2 months after the last weekly infusion. All patients were admitted for acute or acute on chronic renal failure. Renal function improvement was observed in all patients. Adverse effects were mild and mostly reversible. Renal biopsy was repeated in two patients with IgG4-related TIN after 1 year. Histology showed a net reduction of tubulointerstitial infiltration and the disappearance of IgG4-positive plasma cells (Fig. 6) but persistent fibrosis, as in part described by Arai et al. in re-biopsies performed 1-2 months after starting corticosteroid therapy [40].

\section{Conclusions}

IgG4-RD is a fibroinflammatory disorder which can affect any organ. The hallmark histopathological features are dense lymphoplasmacytic infiltrate, storiform fibrosis, and obliterative phlebitis, often accompanied by tissue eosinophilia. Polyclonal CD20+ lymphocytes, T-cells, and IgG4+ plasma cells are prevalent in cellular infiltrates. Elevated serum IgG4 levels are found in the majority of IgG4-RD patients but are not specific. IgG-RKD, i.e. IgG4-RD with renal involvement, may have characteristic imaging findings and different histopathological features such as TIN, MGN and/or RPF. Awareness of the disease is essential for early diagnosis aimed at avoiding irreversible fibrotic processes. 
Glucocorticoid administration is the first effective treatment to be initiated in most patients with acute disease, unless there are major contraindications to their use. An incomplete or unsustained response to glucocorticoids is not infrequent and glucocorticoids have many serious adverse effects in elderly patients. Therefore, more effective and safer therapies are needed. B-cell depletion may play a substantial role in IgG4-RD patients, especially in patients with multiorgan disease and renal involvement.

\section{References}

1. Takahashi H, Yamamoto M, Suzuki C et al (2010) The birthday of a new syndrome: IgG4-related diseases constitute a clinical entity. Autoimmun Rev 9:591-594

2. Stone JH, Khosroshahi A, Deshpande V et al (2012) Recommendations for the nomenclature of IgG4-related disease and its individual organ system manifestations. Arthritis Rheum 64:3061-3067

3. Stone JH, Zen Y, Deshpande V (2012) IgG4-related disease. N Engl J Med 366:539-551

4. Mahajan VS, Mattoo H, Deshpande V et al (2014) IgG4-related disease. Annu Rev Pathol 9:315-347

5. Kamisawa T, Zen Y, Pillai S, Stone JH (2015) IgG4-related disease. Lancet 385:1460-1471

6. Kawano M, Saeki T (2015) IgG4-related kidney disease — an update. Curr Opin Nephrol Hypertens 24:193-201

7. Deshpande V, Zen Y, Chan JK et al (2012) Consensus statement on the pathology of IgG4-related disease. Mod Pathol 25:1181-1192

8. Cortazar FB, Stone JH (2015) IgG4-related disease and the kidney. Nat Rev Nephrol 11:599-609

9. Brito-Zero'n P, Ramos-Casals M, Bosch X, Stone JH (2014) The clinical spectrum of IgG4-related disease. Autoimmun Rev 13:1203-1210

10. Yamamoto M, Takahashi H, Shinomura Y (2014) Mechanisms and assessment of IgG4-related disease: lessons for the rheumatologist. Nat Rev Rheumatol 10:148-159

11. Zen Y, Nakanuma Y (2011) Pathogenesis of IgG4-related disease. Curr Opin Rheumatol 23:114-118

12. Watanabe T, Yamashita K, Fujikawa S et al (2012) Involvement of activation of toll-like receptors and nucleotidebinding oligomerization domain-like receptors in enhanced IgG4 responses in autoimmune pancreatitis. Arthritis Rheum 64:914-924

13. Takeuchi M, Sato Y, Ohno K et al (2014) T helper 2 and regulatory T-cell cytokine production by mast cells: a key factor in the pathogenesis of IgG4-related disease. Mod Pathol 27:1126-1136

14. Kawamura E, Hisano S, Nakashima $\mathrm{H}$ et al (2015) Immunohistological analysis for immunological response and mechanism of interstitial fibrosis in IgG4-related kidney disease. Mod Rheumatol 25:571-578 15. Pradhan D, Pattnaik N, Silowash R, Sk Mohanty (2015) IgG4- related kidney disease - a review. Pathol Res Pract 211:707-711

16. Saeki T, Nishi S, Imai N et al (2010) Clinicopathological characteristics of patients with IgG4-related tubulointerstitial nephritis. Kidney Int 78:1016-1023

17. Raissian Y, Nasr HS, Larsen CP et al (2011) Diagnosis of IgG4- related tubulointerstitial nephritis. J Am Soc Nephrol 22:1343-1352

18. Saeki T, Kawano M (2014) IgG4-related kidney disease. Kidney Int 85:251-257

19. Kawano M, Saeki T, Nakashima H et al (2011) Proposal for diagnostic criteria for IgG4-related kidney disease. Clin Exp Nephrol 15:615-626

20. Alexander MP, Larsen CP, Gibson IW et al (2013) Membranous glomerulonephritis is a manifestation of IgG4related disease. Kidney Int 83:455-462

21. Stone JH (2013) IgG4: a tantalizing link between causes of membranous glomerulonephritis and systemic disease.

Kidney Int 83:348-350

22. Stone JR (2011) Aortitis, periaortitis, and retroperitoneal fibrosis, as manifestations of IgG4-related systemic disease. Curr Opin Rheumatol 23:88-94

23. Khosroshahi A, Carruthers MN, Stone JH et al (2013) Rethinking Ormond's disease: “idiopathic” retroperitoneal fibrosis in the era of IgG4-related disease. Medicine (Baltimore) 92:82-91

24. Fukura H, Taniguchi Y, Matsumoto M et al (2014) IgG4-related tubulointerstitial nephritis accompanied with cystic formation. BMC Urol 14:54-58

25. Soliotis F, Mavragani CP, Plastiras SC et al (2014) IgG4-related disease: a rheumatologist's perspective. Clin Exp Rheumatol 32:724-727

26. Stone JH, Brito-Zero'n P, Bosch X, Ramos-Casals M (2015) Diagnostic approach to the complexity of IgG4-related disease. Mayo Clin Proc 90:927-939

27. Wallace ZS, Deshpande V, Mattoo H et al (2015) IgG4-related disease: clinical and laboratory features in 125 patients. Arthritis Rheumatol 67:2466-2475 
28. Boonstra K, Culver EL, de Buy Wenniger LM et al (2014) Serum immunoglobulin G4 and immunoglobulin G1 for distinguishing immunoglobulin G4-associated cholangitis from primary sclerosing cholangitis. Hepatology 59:19541963

29. Carruthers MN, Khosroshahi A, Augustin T et al (2015) The diagnostic utility of serum IgG4 concentrations in IgG4-related disease. Ann Rheum Dis 74:14-18

30. Wallace ZS, Mattoo H, Carruthers M et al (2015) Plasmablasts as a biomarker for IgG4-related disease, independent of serum IgG4 concentrations. Ann Rheum Dis 74:190-195

31. Kim B, Kim JH, Byun JH et al (2014) IgG4-related kidney disease: MRI findings with emphasis on the usefulness of diffusionweighted imaging. Eur J Radiol 83:1057-1062

32. Tan TJ, Ng YL, Tan D et al (2014) Extrapancreatic findings of IgG4-related disease. Clin Radiol 69:209-218

33. Zhang J, Chen H, Ma Y et al (2014) Characterizing IgG4-related disease with 18F-FDG PET/CT: a prospective cohort study. Eur J Nucl Med Mol Imaging 41:1624-1634

34. Khosroshahi A, Stone JH (2011) Treatment approaches to IgG4- related systemic disease. Curr Opin Rheumatol 23:67-71

35. Khosroshahi A, Bloch DB, Deshpande V, Stone JH (2010) Rituximab therapy leads to rapid decline of serum IgG4 levels and prompt clinical improvement in IgG4-related systemic disease. Arthritis Rheum 62:1755-1762

36. Khosroshahi A, Carruthers MN, Deshpande V et al (2012) Rituximab for the treatment of IgG4-related disease: lessons from 10 consecutive patients. Medicine (Baltimore) 91:57-66

37. Carruthers MN, Topazian MD, Khosroshahi A et al (2015) Rituximab for IgG4-related disease: a prospective, openlabel trial. Ann Rheum Dis 74:1171-1177

38. Khosroshahi A, Wallace ZS, Crowe JL et al (2015) International consensus guidance statement on the management and treatment of IgG4-related disease. Arthritis Rheumatol 67:1688-1699

39. Roccatello D, Sciascia S, Rossi D et al (2011) Intensive shortterm treatment with rituximab, cyclophosphamide and methylprednisolone pulses induces remission in severe cases of SLE with nephritis and avoids further immunosuppressive maintenance therapy. Nephrol Dial Transpl 26:3987-3992

40. Arai H, Hayashi H, Takahashi K et al (2015) Tubulointerstitial fibrosis in patients with IgG4-related kidney disease: pathological findings on repeat renal biopsy. Rheumatol Int 35:1093-1101 
Hypertrophic pachymeningitis

Mikulicz's disease (affecting the lacrimal and salivary glands)

Küttner's tumor (affecting the submandibular glands)

Riedel's thyroiditis

Multifocal fibrosclerosis (affecting the orbits, thyroid gland, retroperitoneum, mediastinum, as well as other tissues and organs)

Inflammatory pseudotumor (affecting the orbits, lungs, kidneys, and other organs)

Eosinophilic angiocentric fibrosis (affecting the orbits and upper respiratory tract)

Mediastinal fibrosis

Periaortitis and periarteritis

Inflammatory aortic aneurysm

Retroperitoneal fibrosis (Ormond's disease)

Autoimmune pancreatitis type 1

Idiopathic hypocomplementemic tubulointerstitial nephritis with extensive tubulointerstitial deposits

Sclerosing mesenteritis

Table 1

Conditions previously considered well-defined disorders, now recognized as organ expression of IgG4-RD

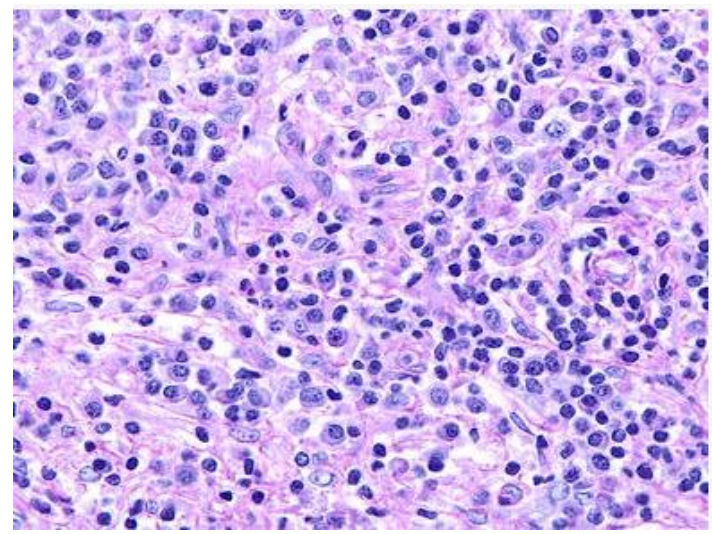

Fig. 1

This renal biopsy from one of our patients with IgG4-related TIN shows a dense interstitial infiltration predominantly composed of plasma cells and lymphocytes. IgG4-related TIN, IgG4-related tubulointerstitial nephritis. 


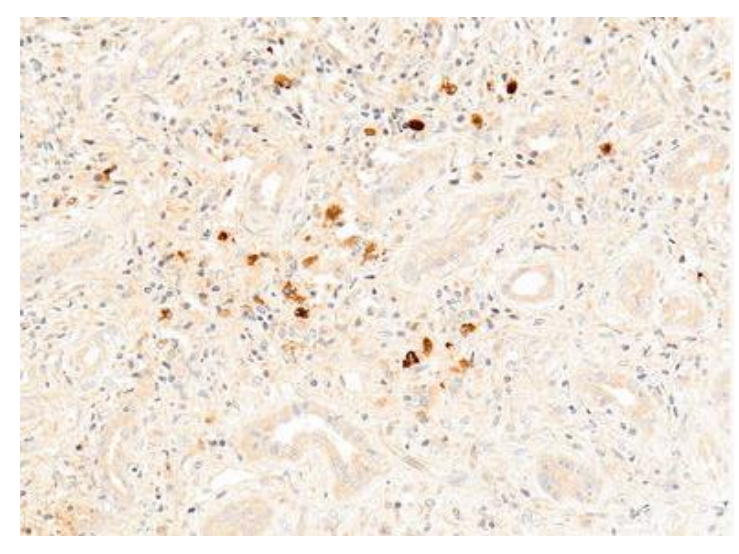

Fig. 2

Renal biopsy from the same patient as in Fig. 1, with IgG4-related TIN, showing more than ten IgG4+ plasma cells/hpf

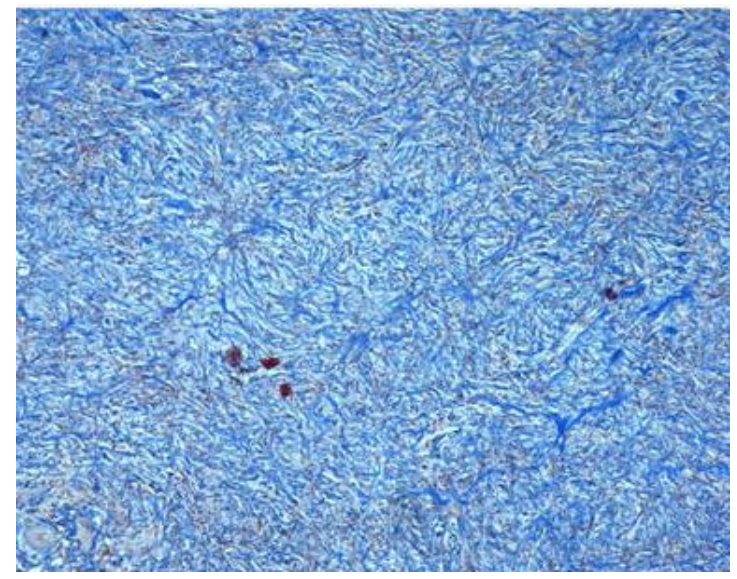

Fig. 3

Specimen of retroperitoneal tissue from one of our patients with IgG4-related RPF, showing the characteristic pattern of storiform fibrosis. IgG4-related RPF, IgG4-related retroperitoneal fibrosis

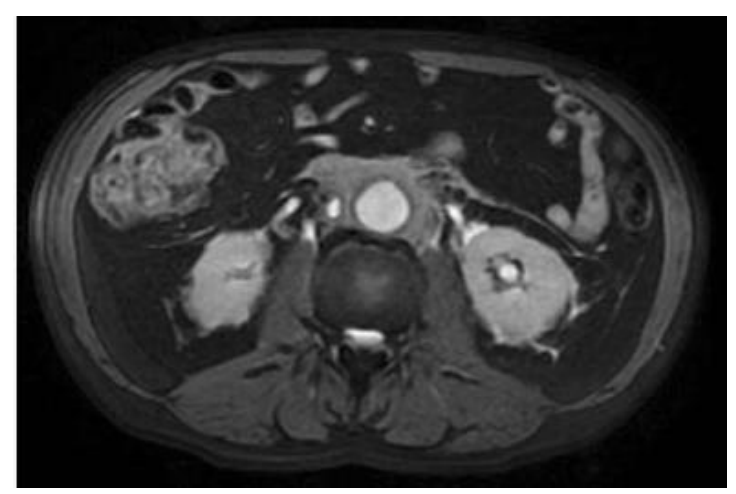

Fig. 4

Contrast-enhanced MRI image of the same patient as in Fig. 3, showing abnormal soft tissue surrounding the abdominal aorta. MRI, magnetic resonance imaging 


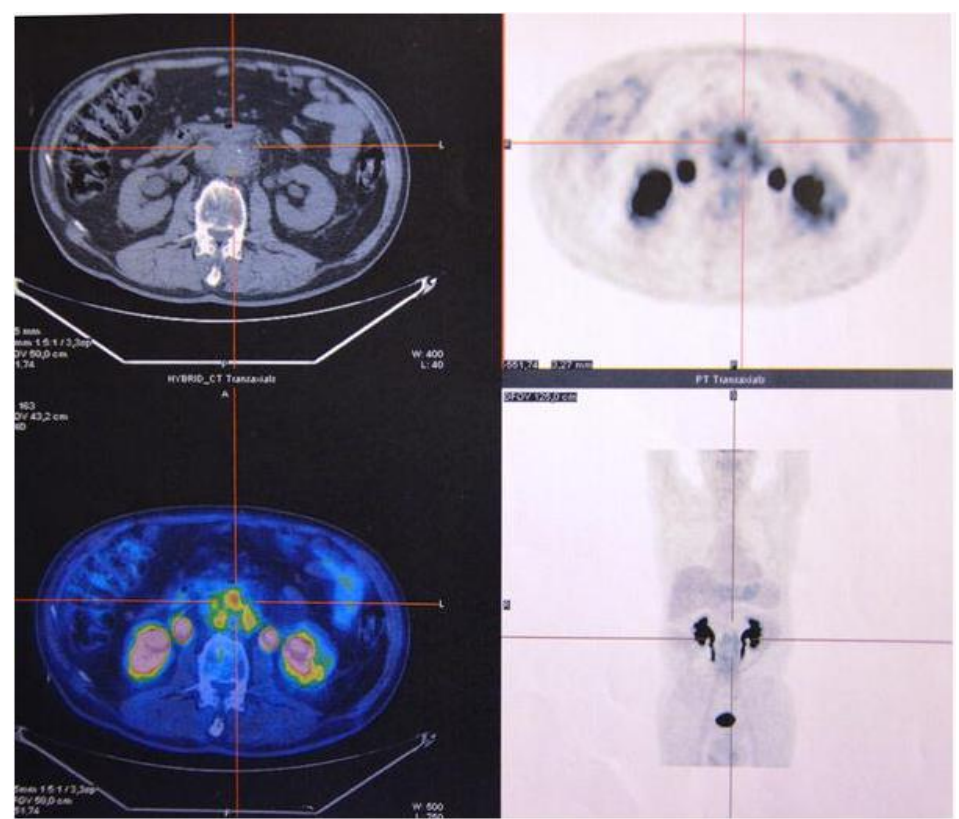

Fig. 5

18F-fluorodeoxyglucose (FDG) positron emission tomography/computed tomography (PET/CT) of the same patient shows retroperitoneal lesions with high metabolic activity

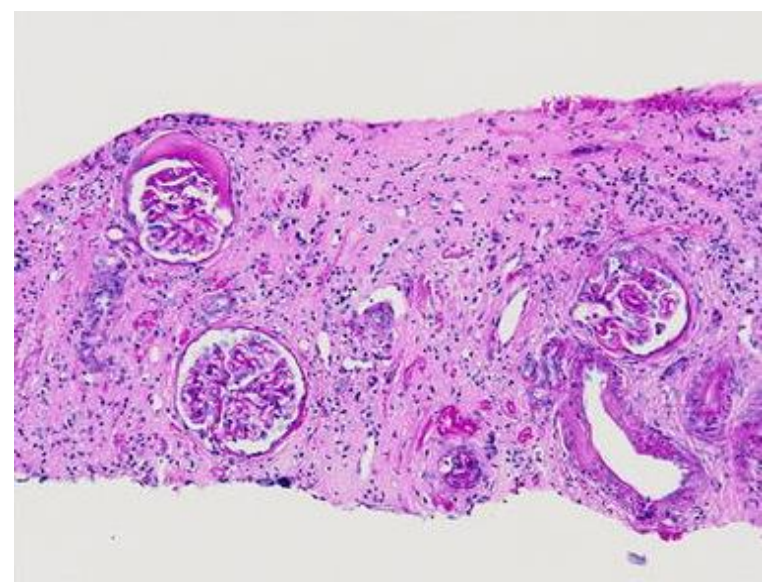

Fig. 6

Repeat renal biopsy in the patient with IgG4-related TIN shows a net reduction of tubulointerstitial infiltration but persistent fibrosis 